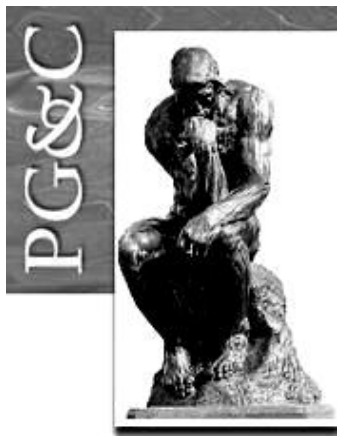

\title{
GESTÃO DO CONHECIMENTO NA ÁREA DE SISTEMAS DE INFORMAÇÕES: UMA REVISÃO NO CONTEXTO BRASILEIRO
}

\author{
Fernanda da Silva Momo \\ Mestre em Administração pela Universidade Federal do Rio Grande \\ do Sul, Brasil. \\ E-mail: fernandamomo@yahoo.com.br \\ Claudia Melati \\ Mestre em Administração pela Universidade Federal do Rio Grande \\ do Sul, Brasil. \\ E-mail: cacaumelati@gmail.com
}

Raquel Janissek-Muniz

Doutora em Administração pela Université Pierre Mendès France de Grenoble, França. Professora da Universidade Federal do Rio Grande do Sul, Brasil.

E-mail: rimuniz@ufrgs.br

\section{Ariel Behr}

Doutor em Administração pela Universidade Federal do Rio Grande do Sul, Brasil. Professor da Universidade Federal do Rio Grande do Sul, Brasil.

E-mail: ariel.behr@ufrgs.br

\begin{abstract}
Resumo
As organizações absorvem informações ao interagirem com o ambiente que estão inseridas e transformam esses conhecimentos obtidos de forma a guiarem a sua forma de agir. Entendendo a importância da temática de gestão do conhecimento, o presente estudo busca sistematizar as abordagens dadas à temática de gestão do conhecimento no contexto de sistemas de informações. Para tornar isso possível, optou-se por um estudo qualitativo, operacionalizado por uma revisão sobre essa temática no período de 2000 a 2016 dos artigos encontrados na base de dados Periódicos Capes. Assim, através da estratégia de busca estipulada esse artigo teve como objeto de estudo 19 artigos que foram analisados a partir da análise de conteúdo. Dessa forma, a partir das análises realizadas foi possível identificar que todos artigos analisados ressaltam a importância da gestão do conhecimento para as organizações, possibilitando a obtenção de vantagem competitiva às organizações. Além disso, percebese que todos os estudos possuem o entendimento de que o sucesso da gestão do conhecimento em uma organização está correlacionado ao fato do engajamento de todas as pessoas que a compõem.
\end{abstract}

Palavras-chave: Gestão do Conhecimento. Abordagens. Importância da Gestão do Conhecimento.

\section{KNOWLEDGE MANAGEMENT IN THE AREA OF INFORMATION SYSTEMS: A REVIEW IN THE BRAZILIAN CONTEXT}

\begin{abstract}
Organizations absorb information when interacting with the environment where they operate and transform that knowledge obtained in order to guide them in their actions. Understanding the importance of the knowledge management theme, this study aims to systematize the approaches given the knowledge management theme in the context of information systems. To make this possible, we opted for a qualitative study, operated by a review about this topic in the period of 2000 to 2016 of the
\end{abstract}


articles found in the Capes periodicals database. Thus, by the stipulated search strategy this article had as object of study 19 articles that were analyzed from the content analysis. Thereby, from the analysis carried out it was possible to identify that all analyzed articles emphasize the importance of knowledge management to organizations, making it possible to obtain competitive advantage to organizations. Moreover, it is clear that all the studies have the understanding that the success of knowledge management in an organization is correlated with the fact of engagement of all the people who compose it.

Keywords: Knowledge management. Approaches. Importance of Knowledge Management.

\section{INTRODUÇÃO}

O contexto econômico contemporâneo baseia-se na premissa de que "a única certeza é a incerteza" e; portanto, a "fonte certa de vantagem competitiva duradoura é o conhecimento" (TAKEUCHI; NONAKA, 2008, p. 39). Ainda, o conhecimento tem se tornado cada vez mais importante para o crescimento econômico e a produtividade dos países (OECD, 2014).

Assim, é notória a importância da temática do conhecimento nas organizações uma vez que ela pode ser compreendida como um processo amplo e criterioso de identificação, maximização, codificação e compartilhamento do conhecimento estrategicamente relevante para as organizações, se demonstrando um ativo valioso nas organizações por ser um facilitador da formulação estratégica na busca por vantagem competitiva sustentável (TERRA, 2001; AL-AMMARY, 2014).

Complementarmente a essas questões, tem-se o fato de que as organizações absorvem informações ao interagirem com o ambiente em que estão inseridas e, segundo Davenport e Prusak (1998), transformam esse conhecimento obtido de forma a agir a partir de uma combinação desses conhecimentos e suas experiências, regras internas, valores. Portanto, ressalta-se o aspecto dinâmico que envolve a temática da Gestão do Conhecimento uma vez que o ambiente e as circunstâncias são complexos e volúveis.

Desse modo, a gestão do conhecimento pode ser visualizada como um recurso valioso para as organizações. À vista disso, tem-se que não basta obter o conhecimento sobre algo uma vez que somente o saber não impacta diretamente na obtenção de uma vantagem competitiva. Sendo assim, há a necessidade de alia-lo à gestão, pois assim, esse conhecimento pode transformar-se em uma vantagem para a organização (LUCHESI, 2012). Assim, estudar a temática de gestão do conhecimento justifica-se uma vez que se está inserido em um contexto de incerteza em que obter uma vantagem competitiva torna-se uma questão relevante para as organizações.

Sendo assim, tendo em vista a importância da gestão do conhecimento para o cenário organizacional, o presente estudo baseia-se na seguinte questão de pesquisa: Qual é o perfil da pesquisa sobre Gestão do Conhecimento na área de Sistemas de Informações no contexto brasileiro? Assim, o objetivo proposto que irá conduzir a pesquisa é sistematizar as abordagens dadas a temática de gestão do conhecimento no contexto brasileiro de forma a enfatizar o uso desse conceito nas pesquisas, ou seja, almeja-se explicitar o "para quê" da gestão do conhecimento no contexto das publicações acadêmicas brasileiras na área de Sistemas de Informações.

No que se refere aos procedimentos utilizados neste estudo, optou-se por um estudo qualitativo, operacionalizado por uma revisão sobre a temática de gestão do conhecimento em um período temporal que abrange os anos de 2000 a 2016 na base de dados Periódicos Capes.

Perspectivas em Gestão \& Conhecimento, João Pessoa, v. 9, n. 1, p. 138-153, jan./abr. 2019. 
Foram estabelecidas estratégias de buscas que resultaram em uma base de 19 artigos que foram analisados através de análise de conteúdo (BARDIN, 2011).

Assim, este estudo estrutura-se trazendo a presente introdução e, em seguida, na seção 2, os conceitos relacionados a Gestão do Conhecimento. Na seção 3 é apresentado os procedimentos metodológicos adotados na presente pesquisa e, na seção 4 , são apresentadas as análises dos resultados. Por fim, apresenta-se na seção 5 as considerações finais.

\section{GESTÃO DO CONHECIMENTO}

Um artigo deve conter partes pré-textuais (título, autoria, resumo, palavras-chave), partes textuais (introdução, desenvolvimento desdobrado em subitens, e considerações finais apresentando a conclusão do estudo) e as partes pós-textuais, que neste formato restringe-se às referências bibliográficas (obras citadas durante o texto).

O conhecimento é considerado como um recurso econômico importante para obtenção de vantagem competitiva (DRUCKER, 1997; AL-AMMARY, 2014). Segundo Ruggles (1998), ao se ter essa noção com base nas organizações, há consequências em relação à forma como um negócio funciona. Assim, para esse autor, há mudanças profundas e dramáticas na organização que influenciam tudo "desde a estratégia de uma empresa aos seus produtos, a partir dos seus processos para a própria forma como a empresa está organizada" (RUGGLES, 1998, p.80, tradução nossa).

Assim, nesse contexto de mudança de perspectiva organizacional tem-se adotado o termo gestão do conhecimento para "descrever tudo, desde os esforços de aprendizagem organizacional para ferramentas de gerenciamento de banco de dados. No entanto, a gestão do conhecimento é mais do que discurso de vendas" (RUGGLES, 1998, p.80, tradução nossa). Dessa forma, na visão de Ruggles (1998, p.80, tradução nossa), compreende-se que a gestão do conhecimento é uma "abordagem para adicionar ou criar valor, mais ativamente aproveitando o know-how, experiência e julgamento residente dentro e, em muitos casos, fora de uma organização".

Nesse sentido, segundo Gold, Malhotra e Segars (2001, p. 185, tradução nossa), "a velocidade e a natureza dinâmica do mercado criou um incentivo competitivo entre muitas empresas a consolidar e conciliar seus ativos de conhecimento como um meio de criar valor que seja sustentável ao longo do tempo". Dessa forma, observa-se que com o intuito de alcançar tal sustentabilidade competitiva muitas empresas buscam utilizar da gestão do conhecimento. Nessa busca, as organizações acabam, em alguns casos, por apenas produzirem uma "consolidação de dados, mas pouca inovação em produtos e serviços" fazendo com que "o conceito de gestão do conhecimento seja convertido em dúvida" (GOLD; MALHOTRA; SEGARS, 2001, p.185, tradução nossa).

Entendendo, portanto, que cada organização possui uma predisposição para lançamento e manutenção de iniciativas relacionadas a gestão do conhecimento, pode-se compreender que a "identificação e avaliação de condições prévias que são necessárias para o esforço para florescer" é tida como uma "chave para entender o sucesso e o fracasso da gestão do conhecimento nas organizações (GOLD; MALHOTRA; SEGARS, 2001, p.185, tradução nossa). Assim, observa-se a complexidade que permeia a temática de gestão do conhecimento uma vez que ela envolve toda a organização.

Sendo assim, evidencia-se o caráter universal da gestão do conhecimento uma vez que sua aplicação faz "sentido tanto para empresas de setores tradicionais, como para empresas em setores de ponta; para empresas em setores primários, como para empresas em setores manufatureiros ou de serviços" (TERRA, 2005, p. 5). Portanto, segundo a visão de Terra (2005, p. 3), a gestão do conhecimento necessita de um envolvimento de toda organização de forma

Perspectivas em Gestão \& Conhecimento, João Pessoa, v. 9, n. 1, p. 138-153, jan./abr. 2019. 
coordenada em todos os planos, sendo eles: "organizacional e individual; estratégico e operacional; normas formais e informais".

A gestão do conhecimento possibilita, portanto, às organizações, o alcance de diferenciais competitivos e ampliação da capacidade de inovação, uma vez que associa a "capacidade de a empresa gerar conhecimento por meio das atividades de pesquisa e desenvolvimento e mesmo no dia a dia dos negócios" com a criatividade de seus colaboradores (GASPAR et al., 2016, p. 151). Dessa forma, as empresas desenvolvem processos para tratar o conhecimento como insumo e recurso que proporcionará uma maior competitividade a empresa e utilizam-se de tecnologias da informação e comunicação para auxiliar nesse processo (GASPAR et al., 2016).

Para tanto, a gestão do conhecimento é feita por meio de práticas gerenciais aplicadas aos processos de aquisição, criação, armazenamento, compartilhamento, disseminação, utilização e descarte de conhecimentos na empresa. Não obstante, observa-se também a incorporação de novas tecnologias de apoio à gestão, desenvolvimento de produtos e de produção oriundas da tecnologia de informação e comunicação. As práticas de gestão do conhecimento e as novas tecnologias da informação e comunicação buscam capacitar a empresa a ser eficaz na consecução de seus objetivos, agregando valor aos produtos e serviços ofertados aos clientes e capacitando-a assim a enfrentar a concorrência. (GASPAR et al., 2016, p. 152)

Assim, ainda com relação às tecnologias de informação no processo de gestão do conhecimento, tem-se elas como ferramentais que "podem ser usadas para sistematizar, melhorar e agilizar a gestão do conhecimento em larga escala de intra e inter firma" (ALAVI; LEIDNER, 2000, p. 4, tradução nossa). Logo, o papel da tecnologia da informação na gestão do conhecimento é "fornecer recursos para pesquisa e recuperação de informações para que indivíduos possam ampliar seus conhecimentos pessoais e aplicar isso às necessidades da organização (ALAVI; LEIDNER, 2000, p. 10, tradução nossa). Portanto, de forma mais sintética, tem-se que seu objetivo é de suporte a construção, compartilhamento e aplicação dos conhecimentos nas empresas (ALAVI; LEIDNER, 2000).

Em outro aspecto, Wiig (1997, p. 2, tradução nossa) descreve a noção de 'corpo de conhecimento' comparando-a a um organismo vivo que "com tudo o que é de fluxos e funções que energizam, motivam e revitalizar a empresa e tornam possível para seu funcionamento". Logo, sendo o 'corpo de conhecimento' as pessoas que formam a organização, tem-se uma forte correlação entre seu bem-estar e a "capacidade para operar efetivamente e para competir" (WIIG, 1997, p. 2, tradução nossa). Portanto, tem-se que é uma função da gestão do conhecimento conseguir manter o corpo de conhecimento organizacional engajado de forma a assegurar o bem-estar e a viabilidade a longo prazo da organização (WIIG, 1997).

Nesse sentido, a autora Aggestam (2006), ressalta a interdependência que existe entre a aprendizagem organizacional e a gestão do conhecimento, de tal forma que uma é dependente da outra para o sucesso organizacional. Assim, remete-se novamente a questão da emergência das organizações de serem organizações de aprendizagem. Por fim, a autora reforça esses argumentos ao comparar essa questão com o questionamento para saber quem veio primeiro, se o ovo ou a galinha e, dessa forma, reforça que "é impossível responder a pergunta que ficou em primeiro lugar, e eles são dependentes um do outro para o sucesso. [...] Uma organização que quer se tornar uma organização de aprendizagem deve prestar atenção para ambos e, portanto, deve haver uma mudança de ênfase para a aprendizagem organizacional e a gestão do conhecimento" (AGGESTAM, 2006, p. 295, tradução nossa). 
No que tange ao objetivo da gestão do conhecimento, Alavi e Leidner (2000) reforçam que a gestão do conhecimento não possui o foco de apenas tornar explícito o conhecimento tácito - aquele que, segundo Nonaka (1994) está embasado na experiência, ação e contexto específico. Sendo assim, observa-se que a gestão do conhecimento objetiva primeiramente "avaliar o conhecimento tácito existente e determinar o que que tem a maioria de valor antes de tentar torná-lo explícito" (ALAVI; LEIDNER, 2000, p. 16, tradução nossa). Nota-se, portanto, que a gestão do conhecimento vai além de explicitar conhecimentos implícitos ela possui o papel de gerenciamento de que conhecimento possuí valor para ser explicitado.

Portanto, nota-se que a gestão do conhecimento é vislumbrada como um recurso estratégico importante nas empresas, nesse sentido, destaca-se o fato de que não é apenas o saber que possibilitará essa vantagem estratégica a organização, mas sim a relação do saber com a gestão desse conhecimento (LUCHESI, 2012). Assim, o desafio a ser enfrentado pelas empresas é a "criação e implantação de processos que gerenciem, armazenem e disseminem o conhecimento" na organização (LUCHESI, 2012, p. 2). Torna-se, portanto, explícita a importância da gestão do conhecimento e essa não apenas significando a geração e armazenamento de conhecimento, mas como um processo que também difunda e compartilhe o saber na organização para que assim, possa proporcionar a organização um diferencial competitivo.

No que tange à geração do conhecimento, segundo Davenport e Prusak (1998), essa ocorre à medida que há interação entre pessoas nos ambientes. Ainda para esses autores, há cinco formas de ocorrer a geração de conhecimento, são elas: Aquisição, recursos dirigidos, fusão, adaptação e redes do conhecimento (DAVENPORT; PRUSAK, 1998). Em outro aspecto, tendo em consideração que o conhecimento é criado a partir da interação entre conhecimentos explícitos e tácitos, Nonaka e Takeuchi (1997) propuseram quatro modos de conversão do conhecimento: Socialização, Externalização, Combinação e Internalização. Assim é por meio desses modos de conversão que o conhecimento se amplia e dissemina.

Dessa forma, posteriormente a uma breve elucidação à questão da relevância da Gestão do Conhecimento e algumas perspectivas relacionadas a essa temática nas organizações, apresenta-se, no Quadro 1, conceitos da Gestão do Conhecimento no entendimento de alguns autores. Torna-se perceptível que apesar das diferenciadas abordagens apresentadas pelos autores o cerne dos conceitos é o mesmo.

Quadro 1 - Conceitos de Gestão do Conhecimento

REFERÊNCIAS

(WIIG, 1997, p. 1, tradução nossa)

(DAVENPORT; LONG; BEERS, 1998, p. 43, tradução nossa)

\section{GESTÃO DO CONHECIMENTO - CONCEITOS}

[...] são os objetivos da gestão do conhecimento (KM): 1. Tornar a empresa agir tão inteligentemente quanto possível para garantir a sua viabilidade e sucesso geral e 2. Caso contrário, realizar o melhor valor de seus ativos de conhecimento. Para alcançar estes objetivos, organizações avançadas construir, transformam, organizam, implantar e utilizar ativos de conhecimento de forma eficaz. Em palavras diferentes, o objetivo geral de KM é maximizar a eficácia de relacionados ao conhecimento da empresa e retorna de seus ativos de conhecimento e para renová-los constantemente. KM é entender, focar e gerenciar o conhecimento sistemático, explícito e deliberado construção, renovação e aplicação. - ou seja, gerenciam os processos de conhecimento efetivo (EKP).

Conhecimento é informação combinada com experiência, contexto, interpretação e reflexão. É uma forma de alto valor da informação que está pronta para aplicar as decisões e ações. Enquanto o conhecimento e a informação podem ser difíceis distinguir às vezes, ambos são mais valiosos e envolvem a participação de mais humana do que os dados brutos sobre o qual podemos ter esbanjavam informatização durante os últimos quarenta anos. 


\begin{tabular}{|c|c|}
\hline & $\begin{array}{l}\text { Dada a importância de tal um ativo, não é surpreendente que as organizações } \\
\text { em todos os lugares estão prestando atenção ao conhecimento - explorando o } \\
\text { que é e como criar, transferir e usá-lo de forma mais eficaz. Gestão do } \\
\text { conhecimento em particular, recentemente floresceu. }\end{array}$ \\
\hline $\begin{array}{c}\text { (RUGGLES, 1998, p.80, } \\
\text { tradução nossa) }\end{array}$ & $\begin{array}{l}\text { Termo gestão do conhecimento para descrever tudo, desde os esforços de } \\
\text { aprendizagem organizacional para ferramentas de gerenciamento de banco de } \\
\text { dados. No entanto, a gestão do conhecimento é mais do que discurso de } \\
\text { vendas. }\end{array}$ \\
\hline $\begin{array}{c}\text { (ALAVI; LEIDNER, 2000, } \\
\text { p. 10, tradução nossa) }\end{array}$ & $\begin{array}{l}\text { A visão do conhecimento como um objeto ou acesso à informação sugere uma } \\
\text { perspectiva de gestão do conhecimento que se concentra na construção e } \\
\text { gestão de estoques de conhecimento. Visualização do conhecimento como um } \\
\text { processo implica um enfoque sobre o fluxo de conhecimento e processos de } \\
\text { criação, compartilhamento e distribuição do conhecimento. A visão do } \\
\text { conhecimento como um recurso sugere uma perspectiva de gestão de } \\
\text { conhecimento centrada na construção de competências essenciais e } \\
\text { compreender a vantagem estratégica de know-how e a criação de capital } \\
\text { intelectual. }\end{array}$ \\
\hline $\begin{array}{l}\text { (GOLD; MALHOTRA; } \\
\text { SEGARS, 2001, p. 185, } \\
\text { tradução nossa) }\end{array}$ & $\begin{array}{l}\text { Gestão do conhecimento é um empreendimento complexo, envolvendo o } \\
\text { desenvolvimento de estruturas que permitem que a empresa a reconhecer, } \\
\text { criar, transformar e distribuir conhecimento. }\end{array}$ \\
\hline (TERRA, 2005, p. 2) & $\begin{array}{l}\text { A Gestão do Conhecimento vai, no entanto, muito além, do investimento em } \\
\text { tecnologia ou o gerenciamento da inovação. } \\
\text { A Gestão do Conhecimento nas organizações passa, necessariamente, pela } \\
\text { compreensão das características e demandas do ambiente competitivo e, } \\
\text { também, pelo entendimento das necessidades individuais e coletivas } \\
\text { associadas aos processos de criação e aprendizado. }\end{array}$ \\
\hline (LUCHESI, 2012, p. 5) & $\begin{array}{l}\text { Gestão do Conhecimento é o processo sistemático de identificação, criação, } \\
\text { renovação e aplicação dos conhecimentos que são estratégicos na vida de uma } \\
\text { organização. É administração dos ativos de conhecimento. Permite à } \\
\text { organização saber o que ela sabe. }\end{array}$ \\
\hline $\begin{array}{c}\text { (GASPAR et al., 2016, p. } \\
\text { 151) }\end{array}$ & $\begin{array}{l}\text { A gestão do conhecimento baseia-se em práticas e processos estruturados para } \\
\text { melhor gerir conhecimento como um recurso. Associadas às práticas, também } \\
\text { se verificam as ferramentas de tecnologia da informação e comunicação que } \\
\text { viabilizam os processos de gestão do conhecimento. }\end{array}$ \\
\hline
\end{tabular}

Fonte: elaborado pelos autores

Sendo assim, pode-se visualizar a Gestão do Conhecimento como um exercício de reflexão, pois conforme Luchesi $(2012$, p. 10) "o tema central da gestão do conhecimento é aproveitar os recursos que já existem na organização para que as pessoas procurem, encontrem e empreguem as melhores práticas em vez de tentar criar algo que já havia sido criado". Portanto, tem-se que a gestão do conhecimento se refere a exploração dos conhecimentos tácitos de forma a torna-los explícitos, sendo que deve existir um filtro de quais conhecimentos são relevantes para a organização nesse processo.

Em vista disso, entende-se que a Gestão do Conhecimento é um tema relevante para as organizações ao passo que pode conferir-Ihes vantagem competitiva. Além disso, destaca-se a interdisciplinaridade desse tema uma vez que o conhecimento é uma questão intrínseca das pessoas e essas compõem as organizações (GASPAR et al., 2016; LUCHESI, 2012). Dessa forma, após compreender-se o contexto ao qual o presente estudo se insere, apresenta-se os métodos utilizados para a realização desta pesquisa. 


\section{METODOLOGIA}

Em relação aos procedimentos utilizados nesta pesquisa, o presente estudo enquadrase como uma investigação de natureza qualitativa, pois possui como objetivo, segundo Oliveira (2011), analisar com profundidade situações particulares ou complexas. Dessa forma, optou-se pela realização de uma revisão sobre gestão do conhecimento e a partir da análise de conteúdo (BARDIN, 2011), buscou-se identificar abordagens, temas e contribuições para esse assunto nos artigos selecionados no contexto brasileiro.

Sendo assim, compreende-se a revisão da literatura como uma revisão de fontes secundárias em que considera resultados e contribuições das pesquisas para um tópico previamente determinado mediante a aplicação de métodos explícitos de busca (LAMB, 2013; LINDE; WILLICH, 2003). Destaca-se que a revisão de literatura realizada se assemelha à metaanálise por também ter como base dados bibliográficos, porém se difere ao passo que não está comprometida com os modelos dessas pesquisas, mas com seus diversos conteúdos de forma mais ampla, identificando as abordagens, temas e contribuições destes trabalhos para a área de estudo. Portanto, compreendendo a revisão como um processo transparente, explícito e reprodutível (MUÑOS, 2009), apresenta-se na Tabela 1 a estratégia de pesquisa adotada para o presente estudo.

Tabela 1 - Estratégia de pesquisa adotada

\begin{tabular}{|c|c|}
\hline REVISÃO & PRÁTICAS ADOTADAS \\
\hline PERÍODO A SER ANALISADO & $2000-2016$ \\
\hline BASES DE DADOS CONSULTADA & Artigos constantes nas bases de dados do Periódicos Capes. \\
\hline CRITÉRIOS DE PESQUISA & $\begin{array}{l}\text { Artigos que contenham no título o termo 'Gestão do Conhecimento' e } \\
\text { o termo 'Gestão do Conhecimento' como assunto do texto. Sendo } \\
\text { utilizado o seguinte filtro na pesquisa: artigos revisados por pares. }\end{array}$ \\
\hline $\begin{array}{c}\text { EXCLUSÕES A SEREM } \\
\text { REALIZADAS }\end{array}$ & $\begin{array}{c}\text { Artigos que, mesmo contendo o critério de busca selecionado, não } \\
\text { tenha relação com o tema de requisitos de software e artigos que } \\
\text { apareceram repetidamente nos resultados. Além disso, selecionou-se } \\
\text { apenas artigos publicados em periódicos acadêmicos que estejam } \\
\text { escritos na língua portuguesa, uma vez que se busca um perfil das } \\
\text { publicações no Brasil. }\end{array}$ \\
\hline ANÁLISE DOS DADOS & $\begin{array}{l}\text { Análise de conteúdo, considerando a categorização de informações } \\
\text { que mais se destacaram nas publicações encontradas. }\end{array}$ \\
\hline
\end{tabular}

Sendo assim, explicita-se os procedimentos adotadas após a pesquisa realizada a partir dos critérios de pesquisa definidos na Tabela 1. Nesse sentido, ressalta-se que dos 25 artigos obtidos como resposta da busca realizada na base de dados excluiu-se dois artigos por não cumprirem o requisito de estarem escritos em língua portuguesa, dois por não estar relacionado ao tema dessa pesquisa e mais dois que apareceram repetidamente. Assim, a partir da busca na base de dados consultada e seleção dos artigos a serem analisados, tabulouse as informações Título, Autores, Resumo, Ano, Método, Abordagem, Considerações e Periódico em uma planilha eletrônica a fim de facilitar a análise de conteúdo. Dessa forma, no Quadro 2, estão descritos os 19 artigos selecionados a partir dos critérios da Tabela 1 para a realização dessa revisão sobre requisitos de software. 
Quadro 2 - Lista de artigos analisados

TÍTULO

Avaliação da maturidade em gestão do conhecimento em Organizações Mineiras

Gestão do Conhecimento em Organizações Orientadas para Projetos

O Estudo da Influência Simbólica do Líder no Processo de Gestão do Conhecimento

A disciplina de gestão do conhecimento no currículo do curso de Biblioteconomia: a experiência da Universidade Federal do Rio Grande do Sul, Brasil

Modelos de gestão do conhecimento em micro e pequenas empresas

Práticas de gestão do conhecimento: o caso da biblioteca universitária da UFSC

Teorias utilizadas nas investigações sobre gestão do conhecimento

Gestão do Conhecimento - GC - como recurso estratégico e fonte de Vantagem Competitiva Sustentável - VCS - na perspectiva Resourcebased view - RBV

Análise dos atributos para avaliação da qualidade da informação nos ambientes de intranet para apoio à gestão do conhecimento

Comunicação como diferencial estratégico na gestão do conhecimento nas organizações

A gestão do conhecimento e a capacidade de competição

Gestão do conhecimento em equipe de vendas multinacional

Gestão do conhecimento e temas relacionados: uma análise através da base Library and Information Science Abstracts - LISA

A propriedade intelectual com elemento estratégico da gestão do conhecimento

A tecnologia de informação como ferramenta de auxílio à gestão da informação e do conhecimento: um estudo de caso do prc programa de repasse do conhecimento no setor elétrico

A gestão do conhecimento: dois casos singulares

A dinâmica da criação e gestão do conhecimento: um estudo de caso

Gestão do conhecimento nas Organizações ou do desconhecimento da realizada organizacional?

Gestão do conhecimento de marketing: uma

\section{AUTORES}

PERIÓDICO

(ZIVIANI; FERREIRA; SILVA, Revista Eletrônica de 2015)

(SHINODA; MAXIMIANO; SBRAGIA, 2015)

Estratégias e Negócios

Gestão de Projetos - GeP

(SCHREIBER, 2015)

Revista Gestão \& Tecnologia

(TEIXEIRA, M. Do R. F., 2014)

Revista Brasileira de Educação em Ciência da Informação

(MOTA; TARGINO, 2013)

Brazilian Journal of Information Science

Revista ACB:

(BEM; AMBONI, 2013)

Biblioteconomia

RISTI: Revista Ibérica de

(XAVIER; OLIVEIRA, M.;

TEIXEIRA, E. K., 2012)

Sistemas e Tecnologias de Informação

(CASTRO; JÚNIOR; PINTO, Revista Organizações em 2012) Contexto

(TRINDADE; OLIVEIRA, M.; BECKER, 2011)

REAd: Revista Eletrônica de Administração

(CAMATTI; FACHINELLI, 2010)

Conexão: Comunicação e Cultura

(SANTOS, A. E. M.; POPADIUK, 2010)

Contextus

(SILVEIRA; LANÇA, 2009)

(MIRANDA; FACHIN;

SANTOS, R. N. M. Dos, 2009)

(CARVALHO; VERAS, 2008)

Revista de Administração FACES Journal

BIBLOS: Revista do Instituto

de Ciências Humanas e da Informação

Revista Eletrônica de Estratégias e Negócios

\begin{tabular}{c|c}
\hline $\begin{array}{c}\text { (LEHMKUHL; VEIGA; } \\
\text { RADOS, 2008) }\end{array}$ & $\begin{array}{c}\text { BIBLOS: Revista do Instituto } \\
\text { de Ciências Humanas e da } \\
\text { Informação }\end{array}$ \\
\hline (VIDAL et al., 2006) & RECADM \\
\hline (MACHADO, 2006) & $\begin{array}{c}\text { Revista de Administração } \\
\text { FACES Journal }\end{array}$ \\
\hline (SANTOS, F. F. Dos et al., & $\begin{array}{c}\text { Revista de Administração } \\
\text { FACES Journal }\end{array}$ \\
\hline (GONÇALVE FILHO, 2003) & Revista de Administração \\
\hline
\end{tabular}

Perspectivas em Gestão \& Conhecimento, João Pessoa, v. 9, n. 1, p. 138-153, jan./abr. 2019. 
Fonte: elaborado pelos autores

Dessa forma, após a elucidação da metodologia utilizada, estratégias de busca e artigos selecionados para a realização desta pesquisa, apresenta-se as análises dos resultados e as considerações finais alcançadas sobre a temática de gestão do conhecimento.

\section{ANÁLISE DOS RESULTADOS}

No que tange a análise dos 19 artigos selecionados, tem-se que a maior quantidade publicada em algum ano foi de 3 artigos e ocorreu no ano de 2015. Sendo assim, observa-se uma pulverização das publicações do tema de gestão do conhecimento na área de Sistemas de Informações; portanto, notou-se que não há um periódico ou autor que concentra a maioria das publicações nobre essa temática. A Tabela 2 apresenta as informações de quantos artigos foram publicados em cada ano a partir da busca realizada e os grupos de análises montados para a apresentação das análises.

Tabela 2 - Grupos de Análises

\begin{tabular}{|c|c|c|c|}
\hline \multicolumn{2}{|c|}{ Grupo de Análise 1} & \multicolumn{2}{|c|}{ Grupo de Análise 2} \\
\hline Ano & Quantidade & Ano & Quantidade \\
\hline 2003 & 1 & 2011 & 1 \\
\hline 2005 & 1 & 2012 & 2 \\
\hline 2006 & 2 & 2013 & 2 \\
\hline 2008 & 2 & 2014 & 1 \\
\hline 2009 & 2 & 2015 & 3 \\
\hline 2010 & 2 & Total & 09 \\
\hline Total & 10 & & \\
\hline \multicolumn{3}{|c|}{ TOTAL GERAL DE ARTIGOS } & 19 \\
\hline
\end{tabular}

Sendo assim, a partir da primeira leitura dos artigos e anotações observadas, preferiuse por apresentar as análises quanto aos grupos de análises criados. Assim, as análises serão apresentadas em duas subseções que serão denominadas Grupo de Análise 1 (contendo os artigos publicados até 2010) e Grupo de Análise 2 (com os artigos publicados de 2011 até 2015) e cada uma dessas seções trará as percepções relativas a 10 e 9 artigos, respectivamente. Essa subdivisão buscou categorizar as abordagens pesquisadas sobre o tema tendo em vista o período de publicação. Ou seja, o que foi publicado até 2010 e como se deu continuidade ou não nos últimos cinco anos analisados.

\subsection{Análise dos artigos}

No que se refere aos artigos publicados até 2010, pode-se perceber que, conforme Tabela 2, divulgou-se 1 artigos em 2003 e 2005 e 2 artigos nos anos de 2006, 2008, 2009 e 2010. Dentro das temáticas abordadas por esses artigos identificou-se assuntos como a interação do conceito de gestão do conhecimento (GC) com a comunicação (CAMATTl; FACHINELLI, 2010); benefícios das práticas de GC associados com a capacidade de competição das organizações (SANTOS, A. E. M.; POPADIUK, 2010); GC na área de vendas de uma empresa multinacional do ramo têxtil (SILVEIRA; LANÇA, 2009).

Outros temas e abordagens são: o que deve ser divulgado e o que deve ser preservado nas organizações em termos da gestão do conhecimento (CARVALHO; VERAS, 2008); uso de 
uma ferramenta de tecnologia da informação para auxílio à gestão do conhecimento (LEHMKUHL; VEIGA; RADOS, 2008); estudo do arcabouço teórico que permeia o conceito de GC (MACHADO, 2006; VIDAL et al., 2006). Além disso, é apresentado um estudo bibliométrico sobre o tema (MIRANDA; FACHIN; SANTOS, 2009) e abordou-se temas sobre validade dos propósitos de GC como ferramenta de intervenção organizacional (SANTOS et al., 2005) e relações entre os processos de GC de marketing, inovação e o desempenho de novos produtos no mercado (GONÇALVE FILHO, 2003).

Tabela 3 - Publicações do Grupo de Análise 1 ARTIGOS

Comunicação como diferencial estratégico na gestão do conhecimento nas organizações (CAMATTI; FACHINELLI, 2010)

A gestão do conhecimento e a capacidade de competição (SANTOS, A. E. M.; POPADIUK, 2010) Gestão do conhecimento em equipe de vendas multinacional (SILVEIRA; LANÇA, 2009)

Gestão do conhecimento e temas relacionados: uma análise através da base Library and Information Science Abstracts - LISA (MIRANDA; FACHIN; SANTOS, R. N. M. Dos, 2009)

A propriedade intelectual com elemento estratégico da gestão do conhecimento (CARVALHO; VERAS, 2008)

A tecnologia de informação como ferramenta de auxílio à gestão da informação e do conhecimento: um estudo de caso do prc - programa de repasse do conhecimento no setor elétrico (LEHMKUHL; VEIGA; RADOS, 2008)

BA gestão do conhecimento: dois casos singulares (VIDAL et al., 2006)

A dinâmica da criação e gestão do conhecimento: um estudo de caso (MACHADO, 2006)

Gestão do conhecimento nas Organizações ou do desconhecimento da realizada organizacional?

(SANTOS, F. F. Dos et al., 2005)

Gestão do conhecimento de marketing: uma pesquisa empírica no setor industrial (GONÇALVES FILHO, 2003)

Fonte: Dados da pesquisa (2018)

Assim, nota-se que esse conjunto de artigos analisados reforça a questão da importância do tema de gestão do conhecimento estar alinhada com a estratégia organizacional e ser um processo que envolve todos na organização. Sendo assim, a abordagem desse tema remete ao que foi mencionado pelos autores no referencial desse artigo (AGGESTAM, 2006; ALAVI; LEIDNER, 2000; GOLD; MALHOTRA; SEGARS, 2001; LUCHESI, 2012; RUGGLES, 1998; TERRA, 2005; WIIG, 1997). Portanto, percebe-se e reforça-se que, apesar dos diversos enfoques dados ao tema de gestão do conhecimento pelos autores do grupo de análise 1 , identifica-se que todos reafirmam a importância desse tema para as organizações. Por fim, apresenta-se a Tabela 4 com o intuito de resumir as abordagens e conclusões dos autores desse grupo de análise (artigos da Tabela 3).

No que se refere aos artigos publicados a partir de 2011 (Tabela 5), pode-se perceber que, conforme Tabela 2, divulgou-se 1 artigo em 2011 e 2014, 2 artigos em 2012 e 2013 e 3 artigos em 2015. Em relação ao entendimento sobre a gestão do conhecimento (GC) nessas publicações, há uma convergência com o que foi apresentado no referencial teórico em que se destaca a relevância da gestão do conhecimento para o alcance de vantagem competitiva (ALAVI; LEIDNER, 2000; DAVENPORT; LONG; BEERS, 1998; GASPAR et al., 2016; GOLD; MALHOTRA; SEGARS, 2001; LUCHESI, 2012; RUGGLES, 1998; TERRA, 2005; WIIG, 1997). Além disso, constata-se que os autores reforçam a necessidade de a gestão do conhecimento estar inserida no contexto estratégico da empresa (ALAVI; LEIDNER, 2000; GOLD; MALHOTRA; SEGARS, 2001; RUGGLES, 1998; TERRA, 2005; WIIG, 1997). 
Tabela 4 - Abordagens da Gestão do Conhecimento do Grupo de Análise 1

\begin{tabular}{|c|c|}
\hline \multirow{10}{*}{$\begin{array}{l}\text { GESTÃO DO } \\
\text { CONHECIMENTO } \\
(\text { GC })\end{array}$} & $\begin{array}{l}\text { Em um cenário em que tudo é único, acredita-se que a gestão do conhecimento tem } \\
\text { como grande aliada a comunicação. (CAMATTI; FACHINELLI, 2010) }\end{array}$ \\
\hline & $\begin{array}{l}\text { Nas empresas estudadas, os gestores reconhecem uma associação entre as práticas } \\
\text { de GC e a aquisição de uma vantagem competitiva. (SANTOS; POPADIUK, 2010) }\end{array}$ \\
\hline & $\begin{array}{l}\text { As empresas podem ter benefícios expressivos com a estruturação da gestão do } \\
\text { conhecimento. (SILVEIRA; LANÇA, 2009) }\end{array}$ \\
\hline & $\begin{array}{l}\text { Observou-se uma maior quantidade de trabalhos sobre gestão do conhecimento na } \\
\text { iniciativa privada do que no setor público. (MIRANDA; FACHIN; SANTOS, } \\
\text { 2009) }\end{array}$ \\
\hline & $\begin{array}{l}\text { Necessidade de entendimento apropriado em busca do equilíbrio entre a proteção } \\
\text { conhecimento e o compartilhamento, de modo útil, considerando indivíduos e } \\
\text { organizações. (CARVALHO; VERAS, 2008) }\end{array}$ \\
\hline & $\begin{array}{l}\text { A utilização da ferramenta PRC (Programa de Repasse de Conhecimento) na } \\
\text { empresa aprimora a consolidação das melhores práticas e fornece subsídios para a } \\
\text { gestão do conhecimento. (LEHMKUHL; VEIGA; RADOS, 2008) }\end{array}$ \\
\hline & $\begin{array}{l}\text { Para a boa prática da GC, as organizações brasileiras deveriam alinhar suas } \\
\text { funções com as informações que dão suporte a tomada de decisão de modo } \\
\text { homogêneo entre todas as áreas envolvidas. (VIDAL et al., 2006) }\end{array}$ \\
\hline & $\begin{array}{l}\text { Empresas brasileiras tendem a buscar modelos estrangeiros e aplicam estes } \\
\text { modelos sem adaptação. Em alguns momentos essa aplicação pode dar resultados } \\
\text { imediatos, mas, para que se configurem como um real aprendizado, é necessário } \\
\text { incorporá-lo não apenas na memória formal da empresa, mas também na mente de } \\
\text { seus colaboradores. (MACHADO, 2006) }\end{array}$ \\
\hline & $\begin{array}{l}\text { Propõem-se um processo de gestão baseado em valores, em substituição ao modelo } \\
\text { gerencial vigente. (SANTOS et al., 2005) }\end{array}$ \\
\hline & $\begin{array}{l}\text { Processos de GC de marketing mostram-se relevantes como geradores de } \\
\text { resultados de novos produtos. (GONÇALVE FILHO, 2003) }\end{array}$ \\
\hline
\end{tabular}
Fonte: Dados da pesquisa (2018)

Sendo assim, os artigos analisados (Tabela 5), versaram sobre a maturidade da gestão do conhecimento em organizações mineiras (ZIVIANI; FERREIRA; SILVA, 2015), processo de gestão de projetos e GC (SHINODA; MAXIMIANO; SBRAGIA, 2015), influência do líder, inovação e GC (SCHREIBER, 2015), importância da GC como disciplina em curso de graduação (TEIXEIRA, M. Do R. F., 2014). Além disso, os artigos abordam a gestão do conhecimento correlacionada a temas como micro e pequenas empresas (MPE) (MOTA; TARGINO, 2013), práticas de compartilhamento de conhecimento em biblioteca universitária (BEM; AMBONI, 2013).

Enfim, nos artigos analisados foram ainda apresentadas as seguintes abordagens: teorias utilizadas em estudos sobre esse tema (XAVIER; OLIVEIRA; TEIXEIRA, 2012), posturas dos gestores em relação a gestão do conhecimento, utilização de medidas e procedimentos relacionados a esse tema e reconhecimento da gestão do conhecimento como fonte de vantagem competitiva (CASTRO; JÚNIOR; PINTO, 2012). Por fim, um dos artigos estudou atributos de qualidade da informação disponibilizada na internet como fonte de suporte a GC (TRINDADE; OLIVEIRA, M.; BECKER, 2011).

Tabela 5 - Publicações do Grupo de Análise 2

\section{ARTIGOS}

Avaliação da maturidade em gestão do conhecimento em Organizações Mineiras (ZIVIANI; FERREIRA; SILVA, 2015)

Gestão do Conhecimento em Organizações Orientadas para Projetos (SHINODA; MAXIMIANO; SBRAGIA, 2015)

O Estudo da Influência Simbólica do Líder no Processo de Gestão do Conhecimento (SCHREIBER, 2015)

A disciplina de gestão do conhecimento no currículo do curso de Biblioteconomia: a experiência da Universidade Federal do Rio Grande do Sul, Brasil (TEIXEIRA, M. Do R. F., 2014)

Perspectivas em Gestão \& Conhecimento, João Pessoa, v. 9, n. 1, p. 138-153, jan./abr. 2019. 
Modelos de gestão do conhecimento em micro e pequenas empresas (MOTA; TARGINO, 2013)

Práticas de gestão do conhecimento: o caso da biblioteca universitária da UFSC (BEM; AMBONI, 2013)

Teorias utilizadas nas investigações sobre gestão do conhecimento (XAVIER; OLIVEIRA; TEIXEIRA, 2012)

Gestão do Conhecimento - GC - como recurso estratégico e fonte de Vantagem Competitiva Sustentável -

VCS - na perspectiva Resource-based view - RBV (CASTRO; JÚNIOR; PINTO, 2012)

Análise dos atributos para avaliação da qualidade da informação nos ambientes de intranet para apoio à gestão do conhecimento (TRINDADE; OLIVEIRA, M.; BECKER, 2011)

Fonte: Dados da pesquisa (2018)

Assim, nota-se uma diversidade de abordagens dadas a temática de gestão do conhecimento nos artigos analisados, mas todos reforçam a ideia da importância desse tema para as organizações. Tendo em vista essas diversas abordagens, apresenta-se a Tabela 6 com o intuito de sumariar todas essas facetas abordadas pelos autores da Tabela 5 em seus artigos.

Tabela 6 - Abordagens da Gestão do Conhecimento do Grupo de Análise 2

Conhecimento e aprendizagem inseridos nos processos e na cultura organizacional,

GESTÃO DO assim, fazem parte da estratégia da organização. (ZIVIANI; FERREIRA; SILVA, CONHECIMENTO 2015)

(GC) Não há melhores práticas de gestão do conhecimento, essas dependerão do contexto organizacional. Práticas em si são relevantes para proporcionar um contexto favorável a GC. (SHINODA; MAXIMIANO; SBRAGIA, 2015)

A atuação do líder sobre a dimensão subjetiva no processo de gestão (maior proximidade da equipe, transparência, participação, troca de experiências) influencia os resultados obtidos a partir da GC. (SCHREIBER, 2015)

Inserção de Gestão do Conhecimento no currículo contribui para construção de um profissional capaz de enfrentar novos desafios. (TEIXEIRA, 2014)

Inadequação dos modelos de GC as MPE, pois são elaborados visando sempre grandes empresas. (MOTA. TARGINO, 2013)

As comunidades práticas são fundamentais para as empresas na implantação de práticas de GC. (BEM; AMBONI, 2013)

Principais teorias relacionadas a aria de si utilizadas nos estudos de Gestão do Conhecimento: Game Theory, Social Capital Theory, Theory of Planned Behavior, Social Exchange Theory, Dynamic Capabilities e Theory of Reasoned Action. (XAVIER; OLIVEIRA; TEIXEIRA, 2012)

Há o reconhecimento por parte dos gestores da importância da GC, mas não estão fortemente implantados os processos para a efetiva GC, bem como o seu alinhamento estratégico com o plano formal da empresa. (CASTRO; JÚNIOR; PINTO, 2012)

Atributos da qualidade da informação no ambiente de internet como suporte a GC em empresas de desenvolvimento de software: precisão, clareza, relevância, credibilidade, completeza, atualidade, tempestividade, fonte e ordem. (TRINDADE; OLIVEIRA; BECKER, 2011)

Fonte: Dados da pesquisa (2018)

Dessa forma, a partir das análises realizadas nos artigos que compunham os grupos de análise 1 e 2 identificou-se a predominância do entendimento da importância da gestão do conhecimento nas organizações, independentemente do enfoque escolhido pelos autores em suas pesquisas. Assim, tomando essa questão como base, apresenta-se a seguir as considerações relativas a pesquisa realizada.

\section{CONSIDERAÇÕES FINAIS}

O presente artigo alcançou o objetivo da pesquisa e respondeu ao questionamento proposto ao identificar e descrever as abordagens das pesquisas realizadas sobre gestão do

Perspectivas em Gestão \& Conhecimento, João Pessoa, v. 9, n. 1, p. 138-153, jan./abr. 2019. 
conhecimento na área de Sistemas de Informações no contexto brasileiro nos anos de 2000 até 2016 (até o mês de junho de 2016). Sendo assim, ao realizar-se as análises dos artigos selecionados foi possível identificar que, em todos os artigos analisados, é enfatizado a importância da gestão do conhecimento para as organizações.

Observou-se também que há uma diversidade de abordagens dada a essa temática, mas percebe-se que todas possuem o entendimento de que o sucesso da gestão do conhecimento em uma organização está correlacionado ao fato do engajamento de todas as pessoas. Assim, notou-se que muitos dos artigos analisados ressaltaram a necessidade de a gestão do conhecimento estar inserida na cultura da empresa e em sua estratégia. Assim, é possível compreender que a gestão do conhecimento depende de uma sinergia organizacional e que suas práticas devem estar alinhadas ao contexto de cada empresa. Portanto, essa temática pode ser entendida como relevante para a obtenção de diferenciais competitivos para a organização que realiza a gestão do conhecimento.

Por fim, destaca-se que mesmo havendo a predominância do entendimento da importância da gestão do conhecimento nas organizações em todos os artigos, observou-se uma evolução e amadurecimento dos temas de pesquisa nos últimos anos (a partir de 2010). Ou seja, passou-se a dar mais foco, a partir de 2010, a temas como a maturidade, processos, influências de líderes na gestão do conhecimento, análise de casos. Enquanto que antes de 2010 os estudos estavam focados na criação de um corpo teórico sobre o assunto, buscando destacar benefícios da gestão do conhecimento, divulgação de informações, conceitos.

Como limitações dessa pesquisa tem-se além da série temporal escolhida, questões como as sutilezas das escolhas realizadas para definição do objeto de análise que foram os artigos, como por exemplo a base de dados escolhida para pesquisas e palavras-chaves selecionadas. Entretanto, ressalta-se que essas escolhas realizadas não afetaram o rigor aplicado a pesquisa. Tendo em vista essas limitações, sugere-se para estudos futuros a realização de uma revisão que abarque um período de tempo maior, em diversos idiomas e em outras bases de dados que versem sobre o tema de gestão do conhecimento.

\section{REFERÊNCIAS}

AGGESTAM, L. Learning organization or knowledge management - which came first , the chicken or the egg ? Information technology and control, v. 35, 2006.

AL-AMMARY, Jaflah. The strategic alignment between knowledge management and information systems strategy: The impact of contextual and cultural factors. Journal of Information \& Knowledge Management, v. 13, n. 1, p. 1450006, 2014.

ALAVI, M.; LEIDNER, D. Knowledge management and knowledge management systems. Journal of strategic information systems, v. 9, n. 2-3, p. 101-105, 2000.

BARDIN, L. Análise de conteúdo. 70. ed. Lisboa: LDA, 2011.

BEM, R. De; AMBONI, N. De F. Práticas de gestão do conhecimento: o caso da bibioteca universitária da ufsc. Revista ACB, v. 18, n. 1, p. 736-751, 2013.

CAMATTI, T. B.; FACHINELLI, A. C. Comunicação como diferencial estratégico na gestão do conhecimento das organizações. Conexão - comunicação e cultura, v. 9, n. 17, 2010.

CARVALHO, I. M.; VERAS, V. M. A propiedade intelectual como elemento estratégico da gestão do 
conhecimento. Estratégia e negócios, v. 1, n. 2, p. 44-68, 2008.

CASTRO, V. A.; JÚNIOR, E. M.; PINTO, R. D. M. C. Gestão do conhecimento - gc - como recurso estratégico e fonte de vantagem competitiva sustentável - vcs - na perspectiva resource-based view -rbv. Organizações em contexto, v. 8, n. 1985, p. 21-41, 2012.

DAVENPORT, T. H.; LONG, D. W.; BEERS, M. C. Building successful knowledge management projects. Sloan management review, 1998.

DAVENPORT, T. H.; PRUSAK, L. Conhecimento empresarial: como as empresas gerenciam o seu capital intelectual. Rio de Janeiro: Campus, 1998.

DRUCKER, P. F. Managing in a time of great change. [S.I.]: Rutledge, 1997.

GASPAR, M. A. et al. Gestão do conhecimento em empresas atuantes na indústria de software no Brasil: um estudo das práticas e ferramentas utilizadas. Informação \& sociedade: estudos, v. 26, 2016.

GOLD, A. H.; MALHOTRA, A.; SEGARS, A. H. Knowledge management: am organizational capabilities perspective. Journal of management information systems, 2001.v. 18, p. 185-214.

GONÇALVES FILHO, C. Gestão do conhecimento de marketing: uma pesquisa empírica no setor industrial. Faces r. adm., v. 2, n. 1, 2003.

LAMB, D. The uses of analysis: rhetorical analysis, article analysis, and the literature review. Academic writing tutor, 2013.

LEHMKUHL, G. T.; VEIGA, C. R. Da; RADOS, G. J. V. A tecnologia de informação como ferramenta de auxílio à gestão da informação e do conhecimento : um estudo de caso do prc-programa de repasse do conhecimento no setor elétrico. Biblos, v. 22, n. 1, p. 19-29, 2008.

LINDE, K.; WILLICH, S. N. How objective are systematic reviews? differences between reviews on complementary medicine. Journal of the royal society of medicine, v. 96, p. 17-22, 2003.

LUCHESI, E. S. F. Gestão do conhecimento nas organizações. Companhia de Engenharia de Tráfego. MACHADO, D. D. P. N. A dinâmica da criação e gestão do conhecimento : um estudo de caso. Faces $r$. adm., v. 5, p. 56-71, 2006.

MIRANDA, A. C. D.; FACHIN, G. R. B.; SANTOS, R. N. M. Dos. Gestão do conhecimento e temas relacionados: uma análise através da base library and information science abstracts -lisa. Biblos, $v$. 23, n. 1, p. 247-258, 2009.

MOTA, D. A. R.; TARGINO, M. D. G. Modelos de gestão do conhecimento em micro e pequenas empresas. Brazilian Journal of Information Science, v. 7, p. 178-200, 2013.

MUÑOS, D. L. . Estudos empíricos de gestão de conhecimento orientados para sustentabilidade: uma revisão sistemática de literatura de 1998 a 2009. Programa de pós-graduação de engenharia e gestão de conhecimento - Universidade Federal de Santa Catarina, 2009.

Perspectivas em Gestão \& Conhecimento, João Pessoa, v. 9, n. 1, p. 138-153, jan./abr. 2019. 
NONAKA, I. A dynamic theory of organizational knowledge creation. Organization science, 1994. v. 5, p. 14-37, 1994.

NONAKA, I.; TAKEUCHI, H. Criação de conhecimento na empresa: como as empresas japonesas geram a dinâmica da inovação. Rio de Janeiro: Campus, 1997.

OECD. Creative industries in the knowledge economy. Tourism and the creative economy. [S.I.]: Organisation for Economic Co-operation and Development, 2014. p. 31-49. Disponível em: http://www.oecd-ilibrary.org/content/chapter/9789264207875-4-en. Acesso em: 07 jan 2019.

OLIVEIRA, A. B. S. Métodos da pesquisa contábil. São Paulo: Atlas, 2011.

RUGGLES, R. The state of the notion: knowledge management in practice. California management review, v. 40, n. 3, 1998.

SANTOS, A. E. M.; POPADIUK, S. A gestão do conhecimento e a capacidade de competição. Contextus - revista contemporânea de economia e gestão, v. 8, p. 23-41, 2010.

SANTOS, F. F. Dos et al. Gestão do conhecimento nas organizações ou do desconhecimetno da realidade organizacional. Faces r. adm., v. 4, n. 2, 2005.

SCHREIBER, D. O estudo da influência simbólica do líder no processo de gestão do conhecimento. Revista gestão \& tecnologia, v. 15, n. 1, p. 200-229, 2015.

SHINODA, A. C. M.; MAXIMIANO, A. C. A.; SBRAGIA, R. Gestão do conhecimento em organizações orientadas para projetos. Gep - revista de gestão e projetos, v. 6, p. 95-110, 2015.

SILVEIRA, M. A. P.; LANÇA, E. C. R. Gestão do conhecimento em equipe de vendas multinacional. Faces r. adm., v. 8, n. 3, p. 41-57, 2009.

TAKEUCHI, H.; NONAKA, I. Gestão do conhecimento. São Paulo: Artmed Editora S.A., 2008.

TEIXEIRA, M. Do R. F. A disciplina de gestão do conhecimento no currículo do curso de biblioteconomia : a experiência da universidade federal do rio grande do sul, brasil. Rebecin - Revista brasileira de educação em ciência da informação, v. 1, n. 1, p. 48-57, 2014.

TERRA, J. C. C. Gestão do conhecimento: o grande desafio empresarial. 3. ed. São Paulo: Negócio Editora, 2001.

TERRA, J. C. C. Gestão do conhecimento: o grande desafio empresarial! Terra forum, p. 1-6, 2005.

TRINDADE, A. L. B.; OLIVEIRA, M.; BECKER, G. V. Análise dos atributos para avaliação da qualidade da informação nos ambientes de intranet para apoio à gestão do conhecimento. Read, v. 70, n. 3, p. 776-801, 2011.

VIDAL, P. et al. A gestão do conhecimento: dois casos singulares. Revista eletrônica de ciência administrativa, v. 5, n. 1, 2006.

WIIG, K. M. Knowledge management: where did it come from and where will it go? Expert systems with applications, 1997. v. 13, n. 1, p. 1-14., 1997.

Perspectivas em Gestão \& Conhecimento, João Pessoa, v. 9, n. 1, p. 138-153, jan./abr. 2019. 
XAVIER, L. A. O. P.; OLIVEIRA, M.; TEIXEIRA, E. K. Teorias utilizadas nas investigações sobre gestão do conhecimento. Risti - revista iberica de sistemas e tecnologias de informação, v. 2012, n. 10, p. 324330, 2012.

ZIVIANI, F.; FERREIRA, M. A. T.; SILVA, S. M. Da. Avaliação da maturidade em gestão do conhecimento em organizações mineiras. Reen - revista eletrônica de estratégia \& negócios, v. 8, n. 1, 2015. 